\title{
BIOSYNTHESIS OF SELENIUM NANOPARTICLES USING KLEBSIELLA PNEUMONIAE AND THEIR RECOVERY BY A SIMPLE STERILIZATION PROCESS
}

\section{Parisa Jafari Fesharaki ${ }^{1,3}$; Pardis Nazari ${ }^{1}$; Mojtaba Shakibaie ${ }^{1}$; Sassan Rezaie ${ }^{2}$; Maryam Banoee ${ }^{3}$ Mohammad Abdollahi" ; Ahmad Reza Shahverdi ${ }^{1^{*}}$}

${ }^{1}$ Department of Pharmaceutical Biotechnology and Pharmaceutical Sciences Research Centre, Faculty of Pharmacy, Tehran University of Medical Sciences, P.O.Box:14155/6451, Tehran, Iran; ${ }^{2}$ Department of Medical Mycology and Parasitology, School of Public Health, Tehran University of Medical Sciences, Tehran, Iran; ${ }^{3}$ Sciences and Research Center, Azad University. Tehran, Iran; ${ }^{4}$ Pharmaceutical Sciences Research Center, Faculty of Pharmacy, Tehran University of Medical Sciences Tehran, Iran.

Submitted: September 17, 2008; Returned to authors for corrections: April 10, 2009; Approved: September $28,2009$.

\begin{abstract}
The use of biologically derived metal nanoparticles for various proposes is going to be an issue of considerable importance; thus, appropriate methods should be developed and tested for the biological synthesis and recovery of these nanoparticles from bacterial cells. In this research study, a strain of Klebsiella pneumoniae was tested for its ability to synthesize elemental selenium nanoparticles from selenium chloride. A broth of Klebsiella pneumoniae culture containing selenium nanoparticles was subjected to sterilization at $121^{\circ} \mathrm{C}$ and 17 psi for 20 minutes. Released selenium nanoparticles ranged in size from 100 to $550 \mathrm{~nm}$, with an average size of $245 \mathrm{~nm}$. Our study also showed that no chemical changes occurred in selenium nanoparticles during the wet heat sterilization process. Therefore, the wet heat sterilization process can be used successfully to recover elemental selenium from bacterial cells.
\end{abstract}

Key-words: selenium nanoparticles; sterilization; Klebsiella pneumoniae; synthesis; recovery

Today, nano metal particles such as silver and gold have drawn the attention of scientists because of their extensive application to new technologies in chemistry, electronics, medicine, and biotechnology (9). Selenium is also important in this respect (18). In medicine, selenium nanoparticles have been reported to demonstrate high biological activity and low toxicity $(17,21)$.

The production of metal nanoparticles can be achieved through various chemical and biological methods $(3,9)$. Carbon disulphide can partially dissolve metal selenium and enable extraction of this element from bacterial cells or polluted soil and sediments (1). The properties of precipitated selenium particles have mainly been investigated by transmission electron microscopic methods after a solvent extraction process (i.e., by carbon disulphide) or after ultrasonic cell disruption (6). However, the synthesis of nanoparticles using microorganisms and plant extracts has been suggested as a possible green alternative to chemical and physical methods $(4,10)$. In recent years, many different techniques have been described for the biological synthesis of silver and gold metal nanoparticles (10). Although many reports have been previously published about the reduction of selenium

\footnotetext{
*Corresponding Author. Mailing address: Department of Pharmaceutical Biotechnology and Biotechnology Research Center, Faculty of Pharmacy, Tehran University of Medical Sciences, Tehran, Iran.; Tel.: +98 2166482706 Fax: +98 2166461178.; E-mail: Shahverd@sina.tums.ac.ir
} 
oxyanions (selenite and selenate) to elemental selenium under aerobic and anaerobic conditions, the capacity of a large number of bacteria to form selenium nanoparticles has yet to be demonstrated $(13,20)$. In one study, Losi and his coworkers reported that Enterobacter cloacae were able to remove selenium oxyanions from a culture medium by reducing them to elemental selenium particles in a range of sizes $<1 \mu \mathrm{m}(8)$. These reported biological processes have been suggested for the bioremediation and detoxification of selenium-polluted environments $(5,8)$. The main objective of this research was to study another biological method of synthesizing selenium nanoparticles.

In this research, a simple wet analytical technique (a titrimetric method) was first employed for a preliminary evaluation of the reduction potential of Klebsiella pneumoniae (Enterobacteriaceae) grown in various culture media. The reduction capability of this test strain in converting $\mathrm{Se}^{+4}$ to elemental selenium was studied using a specifically chosen culture broth. Moreover, in this investigation, the bacterial cell mass was treated by a wet heat sterilization process in a laboratory autoclave under conventional conditions $\left(121^{\circ} \mathrm{C}, 17\right.$ psi for 20 minutes) and the chemical and morphological properties of the released selenium particles were characterized by Transmission Electron Microscopy (TEM), UV-Visible Spectroscopy and Energy Dispersive Spectroscopy (EDS).

The test strain was Klebsiella pneumonia from our collection (16). The identity of this strain was further confirmed by conventional biochemical methods. Various culture broths (Table 1) from Merck KGaA, of Darmstadt, Germany, were prepared, sterilized, and inoculated with a fresh batch of test strain (K. pneumonia). The culture flasks were incubated for $24 \mathrm{~h}$ at $37^{\circ} \mathrm{C}$. After the incubation period, the cultures were centrifuged at $14000 \mathrm{rpm}(18000 \times \mathrm{g})$ for 15 minutes and their supernatants were used for a titrimetric assay. In this assay the reduction properties of the different supernatants were quantitatively investigated by the conventional potassium permanganate back-titration method. All supernatants $(6 \mathrm{~mL})$ were diluted by distilled water $(20$ $\mathrm{mL}$ ) and acidified with $2 \mathrm{~mL}$ of phosphoric acid (1.5 N). Next, all diluted samples were oxidized with an excess of potassium permanganate $(0.1 \mathrm{~N})$ for 30 minutes at $60^{\circ} \mathrm{C}$, and the unreacted permanganate was titrated with a $0.04 \mathrm{~N}$ oxalic acid solution. The end-point was determined when the solution's violet color, produced by excess potassium permanganate, disappeared. The total concentration of the reduction agents in different supernatants was calculated using the volume and normality of the permanganate sample-oxidizing solution and was reported as $\mathrm{mg}$ of $\mathrm{KMnO}_{4}$ per $\mathrm{ml}$ of the supernatants. The concentration of reduction agents in the various sterile, noninoculated culture media were also evaluated using the potassium permanganate back titration method and subtracted from the total concentration of reduction agents.

Table 1. The reduction ability of the culture media before and after $K$. pneumoniae inoculation and incubation for 24 hours at $37^{\circ} \mathrm{C}$.

\begin{tabular}{|c|c|c|c|}
\hline \multirow[b]{2}{*}{ Culture media } & \multicolumn{2}{|c|}{ Amount of $\mathrm{KMnO}_{4}$ used for oxidation $(\mathrm{mg} / \mathrm{ml})$} & \multirow{2}{*}{$\begin{array}{l}\text { Reduction ability } \\
(\mathrm{mg} / \mathrm{ml})(\mathrm{C})^{1}\end{array}$} \\
\hline & $\begin{array}{l}\text { Culture broth before } \\
\text { inoculation (A) }\end{array}$ & $\begin{array}{l}\text { Culture broth after } \\
\text { incubation with test } \\
\text { strain (B) }\end{array}$ & \\
\hline Müller-Hinton broth & 3.55 & 2.43 & 1.12 \\
\hline Triptic Soy broth & 4.54 & 2.62 & 1.92 \\
\hline Nutrient broth & 2.21 & 1.95 & 0.26 \\
\hline Luria-Bertani broth & 3.36 & 2.40 & 0.96 \\
\hline
\end{tabular}

${ }^{1}$ The reduction ability (C) of the test strain in different culture media after an incubation time (24 hours) was determined by subtracting the data listed in column B from column A. 
Preparation of selenium nanoparticles and determination of residual $\mathrm{Se}^{+4}$ were performed using following method. A uniform inoculum was prepared by aseptically transferring a loopful of K. pneumoniae from a Triptic Soy Agar (TSA) plate to $100 \mathrm{ml}$ of sterile Triptic Soy Broth (TSB) and growing the culture to an OD600 of 1.0. This solution constituted the inoculum. TSB at $\mathrm{pH} 7.2$ was prepared, sterilized, and supplemented with a $200 \mathrm{mg} / 1 \mathrm{Se}^{+4}$ solution (equal to 559.19 $\mathrm{mg}$ of selenium chloride). Subsequently, $1 \%$ (v/v) of the inoculum was added to selenium containing TSB, and the culture flask was incubated at $37{ }^{\circ} \mathrm{C}$ for 24 hours. A control flask containing TSB without selenium chloride was inoculated with a test strain and incubated under the same conditions. A conventional plating technique was used for monitoring bacterial cell concentrations during the incubation period. After incubation, the reduction of the $\mathrm{Se}^{+4}$ ions in the solution was determined by a potassium permanganate back titration method (11). For this propose, an aqueous sample $(6 \mathrm{ml})$ was removed and centrifuged at $14,000 \mathrm{rpm}(18000 \times \mathrm{g})$ for 15 minutes. Next, this supernatant was assayed using the titrimetric analysis previously described. Each $\mathrm{mg}$ of potassium permanganate that was used as an oxidizing agent in the titrimetric analysis can be stoichiometrically represented as

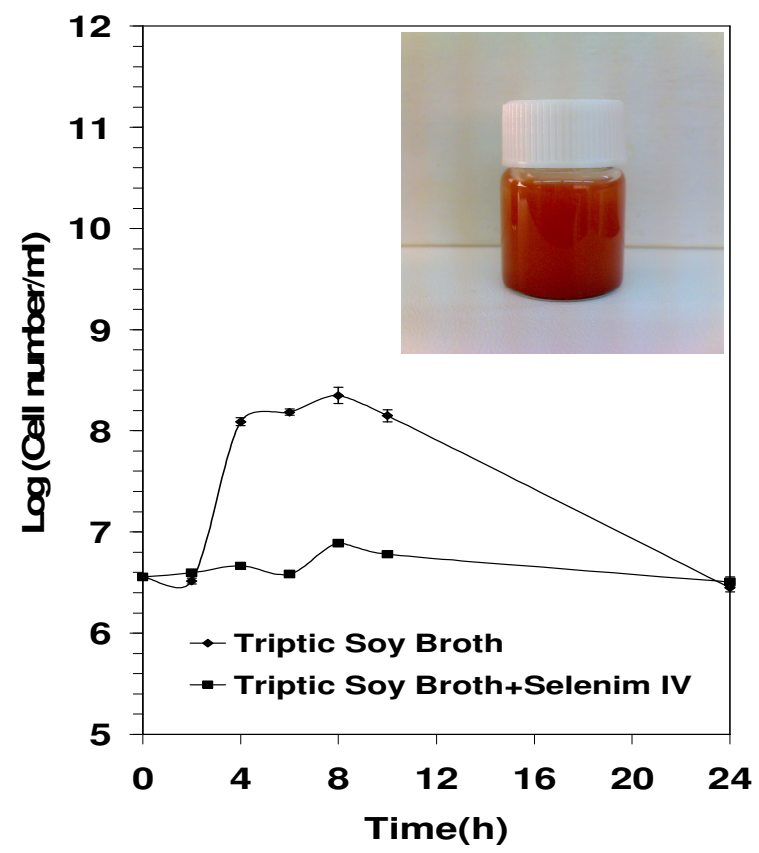

$1.66 \mathrm{mg}$ of $\mathrm{Se}^{+4}$ ions. In the next step, $K$. pneumoniae cells containing the red selenium particles were disrupted using a wet heat sterilization process in a laboratory autoclave at $121^{\circ} \mathrm{C}, 17 \mathrm{psi}$ for 20 minutes. The released selenium nanoparticles were centrifuged at $14000 \mathrm{rpm}(18000 \times \mathrm{g})$ for 15 minutes and washed three times with distilled water. The washed sample was sonicated for 10 minutes (Tecna6, TechnoGaz, Italy) and characterized by transmission electron microscopy (Philips EM400T/FEG), UV-Visible spectroscopy, and energy-dispersive spectroscopy (EDS).

The results are demonstrated in Table 1 and Fig. 1. The reduction properties of different culture supernatants of $K$. pneumoniae were first investigated using an oxidationreduction titrimetric assay involving $\mathrm{KMnO}_{4}$ (Table 1). The culture supernatant of $K$. pneumoniae grown in TSB showed the highest reduction ability among the tested culture supernatants (Table 1). Therefore, this culture media was chosen for the biological synthesis of selenium nanoparticles. The reduction of $\mathrm{Se}^{+4}$ by K. pneumoniae grown in TSB and the formation of selenium nanoparticles were investigated. The appearance of a red color in the culture flasks suggested the formation of elemental selenium (1).

The inset to upper left-hand graph in Fig. 1 shows the

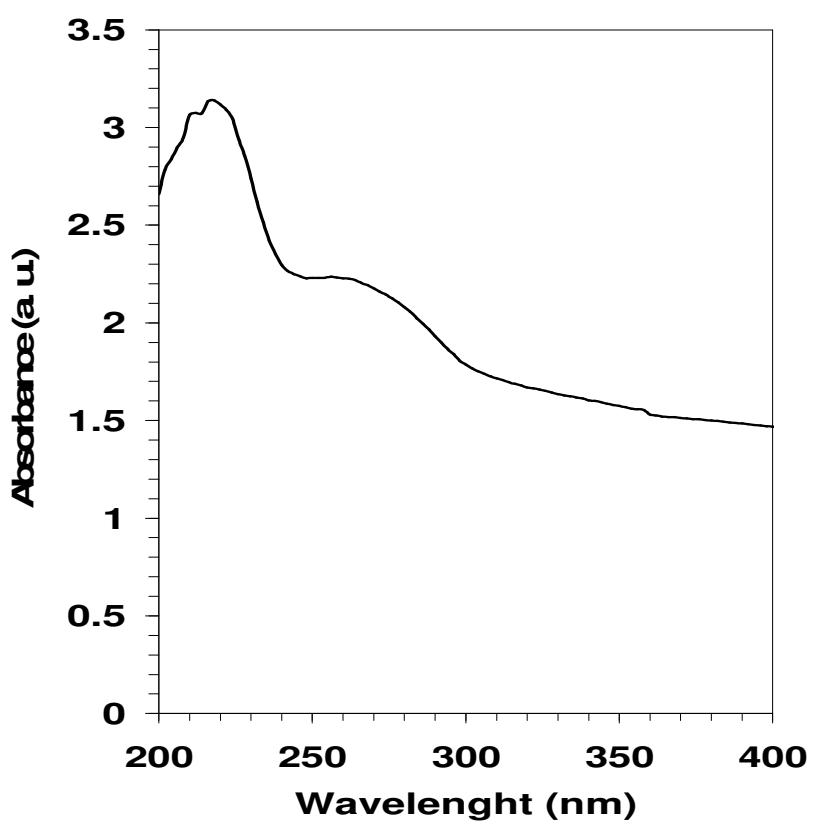



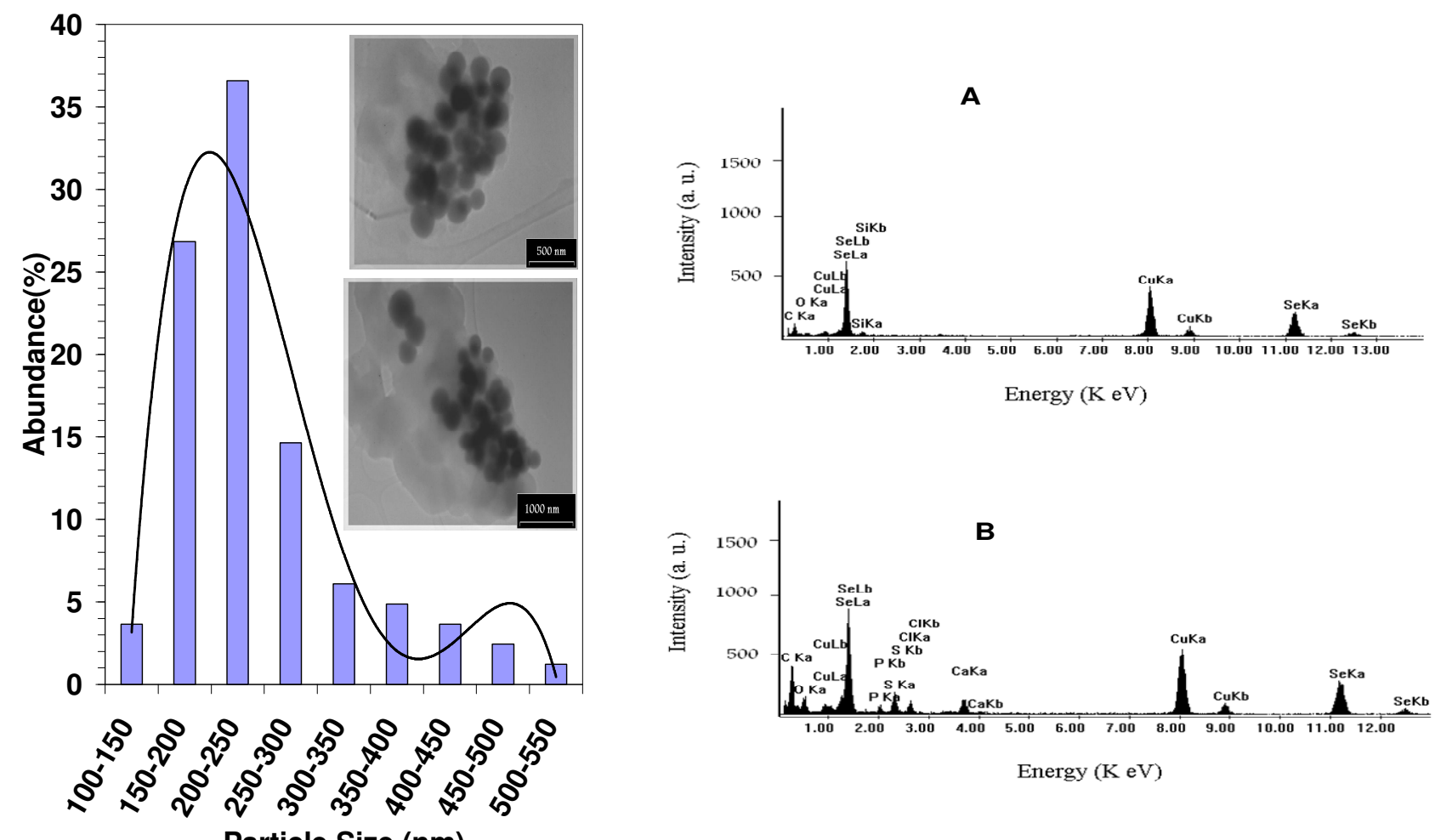

Particle Size (nm)

Energy (K eV)

Figure 1. The upper left hand-graph shows the time course of Klebsiella pneumoniae growth in the presence and absence of $\mathrm{Se}^{+4}$ $(200 \mathrm{mg} / \mathrm{l})$. Triptic Soy broth was used as culture media. The inset in this graph (upper left-hand) demonstrates the container containing the red selenium particles prepared using Klebsiella pneumoniae after incubation period (24 hours). Upper right graph shows the UV-visible spectra of selenium colloid prepared using Klebsiella pneumoniae. Lower left graph demonstrated the related particle size distribution histogram obtained after measuring 350 particles from each sample. Also inset illustrations in this histogram shows transmission electron micrographs of prepared selenium nanoparticles after the sterilization process. EDS spectrum of selenium nanoparticles prepared using Klebsiella pneumoniae before (A) and after (B) the sterilization process are shown in the lower right.

container of selenium colloid prepared using $K$. pneumonia. The upper left-hand graph in Fig.1 also shows the growth profile of $K$. pneumoniae in the absence and presence of $\mathrm{Se}^{+4}$ (200 mg/l). The concentration of viable cells has not changed during incubation in the presence of $\mathrm{Se}^{+4}(200 \mathrm{mg} / \mathrm{l})$. The concentration of residual $\mathrm{Se}^{+4}$ ions in inoculated and uninoculated $\mathrm{Se}^{+4}$-containing TSB was also determined after the incubation period. No selenium reduction was observed in the selenium-supplemented TSB without inoculation (sterile control), and we did not observe any elemental selenium formation in the absence of the test strain. In contrast, the concentration of selenium ions was decreased from 200 to 80 $\mathrm{mg} / \mathrm{l}$ in culture media inoculated with $K$. pneumoniae and incubated for $24 \mathrm{~h}$ at $37^{\circ} \mathrm{C}$.

The culture containing selenium particles was sterilized under the conditions previously mentioned and washed. Selenium nanoparticles were further characterized by UVvisible spectroscopy. The technique outlined above proved to be very useful for the analysis of nanoparticles. As illustrated in upper right spectrum in Fig. 1 strong, absorption bands with a maximum (218, and $248 \mathrm{~nm}$ ) located between 200nm and $300 \mathrm{~nm}$ was observed due to formation of selenium 
nanoparticles produced during reduction of selenium ions $\left(\mathrm{Se}^{+4}\right)(14)$.

The inset photographs to lower left-hand histogram in Fig. 1 show representative TEM images of the selenium nanoparticles synthesized by $K$. pneumoniae and released after sterilization process. The particle size histogram of selenium particles (the lower left-hand illustration in Fig. 1) shows that the particles ranged in size from 100 to $550 \mathrm{~nm}$ and possessed an average size of $245 \pm 82.47 \mathrm{~nm}( \pm \mathrm{SD})$. Our study on the released selenium particles using EDS shows that selenium nanoparticles remained chemically unchanged during the wet heat sterilization process (lower right-hand illustrations). In the analysis of the selenium nanoparticles by EDS, the presence of elemental selenium signals were confirmed (Lower right-hand illustrations). The selenium nanocrystallites displayed optical absorption bands, peaking at $1.5,11.2$, and $12.5 \mathrm{keV}$, which is typical of the absorption of metallic selenium nanocrystallites (12). Therefore, the wet heat sterilization process can be used successfully for recovering elemental selenium from a bacterial culture broth.

In conclusion, Selenium possesses several applications in medicine, chemistry, and electronics. In recent years, there has been an increasing interest in synthesizing metal particles using chemical and biological methods $(7,10,22)$. The use of "green" synthesis of metal nanoparticles is going to be of considerable importance; thus, appropriate methods should be developed and tested, especially for the recovery of these nanoparticles from natural resources such as bacterial cells. In the present research, an oxidation-reduction titrimetric assay involving $\mathrm{KMnO}_{4}$ was first used for determination of the reduction properties of different culture supernatants of K. pneumoniae. The highest reduction ability was observed for the culture supernatant of $K$. pneumoniae grown in TSB. Therefore, this culture media was chosen for the biological synthesis of selenium nanoparticles. The bio-recovery of selenium nanoparticles from a selenium chloride supplemented TSB was further investigated by $K$. pneumoniae. A wet heat sterilization process was used for disrupting the bacterial cells containing the selenium particles. The released nanoparticles showed nanoparticles in the range of $100-550 \mathrm{~nm}$, with an average size of $245 \mathrm{~nm}$. These nanoparticles were chemically stable the during sterilization process, suggesting a possible utilization of this process (wet heat sterilization) for recovering selenium nanoparticles from the cell mass of bacteria, or for recovering other intracellular metal nanoparticles generated by microorganisms $(2,15)$. In the other hand the strong EDS signals from the atoms in the nanoparticles confirmed the reduction of selenium ions to elemental selenium and its chemical stability during cell disruption using the sterilization process. To the best of our knowledge, this is the first report on the biogenesis of selenium nanoparticles using Klebsiella pneumoniae and their characterization after undergoing a wet heat sterilization process.

\section{ACKNOWLEDGEMENTS}

This research was financially supported by Pharmaceutical Sciences Research Center, Faculty of Pharmacy, Tehran University of Medical Sciences, Tehran, Iran. We wish to thank from Mr. Hossein Jamalifar for his excellent technical assistance.

\section{REFERENCES}

1. Ahluwalia, G.; Saxena, YR.; Williams, HH. (1968). Quantitative studies on selenite metabolism in Escherichia coli. Arch. Biochem. Biophys., $124,79-84$.

2. Ahmad, A.; Senapati, S.; Khan, MI.; Kumar, R.; Ramani, R.; Srinivas, V.; Sastry, M. (2003). Intracellular synthesis of gold nanoparticles by a novel alkalotolerant actinomycete, Rhodococcus Species. Nanothechnology, 14, 824-828.

3. Ayyub, P.; Chandran, R.; Taneja, R P.; Sharma, A.; Pinto, R. (2001). Synthesis of nanocrystalline material by sputtering and laser ablation at low temperature. Applied. Phys. A., 73, 67-73.

4. Bhattacharya, D.; Rajinder, G. (2005). Nanothechnology and potential of microorganisms. Crit. Rev. Biotechnol., 25, 199-204.

5. Cantafio, A.W.; Hagen, KD.; Lewis, G E.; Bledsoe, T L.; Nunan, K M.; Macy, JM. (1996). Pilot-scale selenium bioremediation of San Joaquin drainage water with thauera selanatis. Appl. Environ. Microb., 62, 32983303 .

6. Gerrard, T L.; Telford, J N.; Williams, HH. (1974). Determination of selenium deposits in Escherichia coli by electron microscopy. J. Bacteriol. 119, 1057-1060. 
7. Liu, M.; Zhang, S.; Shen, Y.; Zhang, ML. (2004). Selenium nanoparticles prepared from reverse microemulsion process. Chinese Chem. Lett., 15, 1249-1252.

8. Losi, ME.; Frankenberger, WT. (1997). Reduction of selenium oxyanions by Enterobacter Cloacea SLD1a-1: isolation and growth of the bacterium and its expulsion of selenium particles. Appl. Environ. Microb., 63, 3079-3084.

9. Mandal, D.; Bolander, ME.; Mukhopadhyay, D.; Sarkar, G.; Mukherjee, P. (2006). The use of microorganisms for formation of metal nanoparticles and their application. Appl. Environ. Microb., 69, 485-492.

10. Mohanpuria, P.; Rana, N K.; Yadav, SK. (2008). Biosynthesis of nanoparticles: technological concepts and future applications. Nanopart. Res., 10, 510-517.

11. Naidu, PP.; Rao, GG. (1970). Differential titrimetric determination of mixture of selenium and tellurium. Indian Academy of Sciences.

12. Oremland, RS.; Herbel, MJ.; Switzer- Blum, J.; Langley, S.; Beveridge, TJ.; Ajayan, PM.; Sutto, T.; Ellis, AV.; Curran, S. (2004). Structural and spectral features of selenium nanospheres produced by Sr-respiring bacteria. Appl. Environ. Microb., 70, 52-60.

13. Oremland, R S.; Hollibaugh, JT.; Maest, A S.; Presser, T S.; Miller, L G.; Culbertson, CW. (1989). Selenate reduction to elemental selenium by anaerobic bacteria in sediments and culture: Biogeochemical significance of a novel, sulfate-independent respiration. Appl. Environ. Microb., 55, 2333-2343.

14. Praharaj, S.; Nath, S.; Panigrahi, S.; Basu, S.; Ghosh, S K.; Pande, S.; Jana, S.; Pal, T. (2006). Room temperature synthesis of coinage metal (Ag, $\mathrm{Cu}$ ) chalcogenides. Chem. Commun., 3836-3838.

15. Senapati, S.; Mandal, D.; Ahmad, A.; Khan, MI.; Sastry, M.; Kumar, R.
(2004). Fungus mediated synthesis of silver nanoparticles: a novel biological approach. Indian J. Phys., 78A, 101-105.

16. Shahverdi, A R.; Minaeian, S.; Shahverdi, HR.; Jamalifar, H.; Nohi, A. (2007). Rapid synthesis of silver nanoparticles using culture supernatants of Enterobacteria: A novel biological approach. Process Biochem., 42, 919-923.

17. Wang, H.; Zhang, J.; Yu, H. (2007). Elemental selenium at nano size possesses lower toxicity without compromising the fundamental effect on selenoenzymes: comparison with selenomethionine in mice. Free Radic. Biol. Med., 42, 1524-1533.

18. Xu, H.; Huang, K. (1994). Chemistry, Biochemistry of Selenium and its Application in Life Science. Hua East University of Science \& Technology Press.

19. Yadav, V.; Sharma, N.; Prakash, R.; Raina, K K.; Bharadwaj, LM.; Tejo Prakash, N. (2008). Generation of selenium containing nano-structures by soli bacterium, Pseudomonas aeruginosa. Biotechnology, 7, 299-304.

20. Yee, N.; Ma, J.; Dalia, A.; Boonfueng, T.; Kobayashi, DY. (2007). Se (VI) Reduction and the precipitation of se (0) by the facultative bacterium Enterobacter cloacae SLD1a-1: are regulated by FNR. Appl. Environ. Microb., 73, 1914-1920.

21. Zhang, J.; Wang, X.; Xu, T. (2007). Elemental selenium at nano size (Nano-Se) as a potential chemopreventive agent with reduced risk of selenium toxicity: comparison with se-methylselenocysteine in mice. Toxicol. Sci., 101, 22-31.

22. Zhang, S.; Zhang, J.; Wang, H.; Chen, H. (2004). Synthesis of selenium nanoparticles in the presence of polysaccharides. Mater. Lett., 28, 25902594. 